\title{
Effects of Comorbidity and Clustering upon Referrals in Primary Care
}

\author{
Frederick M. Chen, MD, MPH, George E. Fryer, Jr., PhD, and Thomas E. Norris, MD
}

Objective: To examine the effect of patient characteristics and comorbidity on referrals in primary care. Methods: Cross-sectional analysis of patient encounters and referrals during a 1-year period for a primary care network of 9 clinics. The analysis adjusted for the clustering effect of physicians and clinics on the data.

Results: 23,720 specialty referrals were generated from 251,240 patient encounters, resulting in a total referral rate of 9.4 referrals per 100 encounters. Age, gender, and certain comorbid conditions were significant predictors of referral for any given encounter.

Conclusions: Patient characteristics and comorbidity are predictors of referral. Studies of primary care processes need to account for clustering of physicians and clinics in their research design. (J Am Board Fam Pract 2005;18:449-52.)

In this era of rising health care costs and increasing emphasis on patient safety, surprisingly little is known about patients' transition from primary to specialty care. Many explanations have been offered for the documented large variations in referral rates. ${ }^{1-3}$ Comorbid conditions and patients' overall health status are clearly associated with the decision to refer, ${ }^{4-6}$ but there is also evidence that referral rates are also related to patient preferences and demographic factors. ${ }^{7,8}$ At another level, attributes of health care provider personnel and facilities, and the systems in which they work have been found to be predictors of patient referral. ${ }^{9-13}$ Utilization re- $^{-}$ view and reimbursement mechanisms are systemic variables that influence referral patterns. ${ }^{14,15}$ The interplay of these multiple factors in the referral process is complex and continues to defy any simple explanation. We focused our analysis on a few critical predictors-patient characteristics and comorbidity-while introducing the use of multilevel analysis in referral research.

Submitted 16 March 2005; revised 7 June 2005; accepted 13 June 2005.

From the Department of Family Medicine, University of Washington, Seattle, WA (FMC, TEN); and Department of Pediatrics, University of Rochester, Rochester, NY (GEF).

Conflict of interest: none declared.

Corresponding author: Frederick M. Chen, MD, MPH, Department of Family Medicine, University of Washington, 4311 11th Avenue NE, Suite 210, Seattle, WA 98195-4982 (e-mail: fchen@u.washington.edu).
There is growing recognition of the importance of multilevel analysis when analyzing clustered and nested data. ${ }^{16-19}$ Failing to account for the multilevel structure of patients, providers, and clinics may underestimate standard errors or result in inefficient estimates. Recognizing that the theoretical model of the referral process is complex and multifactorial, we chose to focus on a few significant predictors of referral. These were patient characteristics, comorbidity, and the clustering effect of primary care data. In this study, we sought to examine the contribution of comorbidity, as measured by individual ambulatory diagnostic groups (ADGs), to physician referral tendencies, and to identify characteristics of patients most likely to be referred. We hypothesized that comorbidity, as measured by ADGs, would have a strong influence on referral likelihood, even after adjusting for clustering.

\section{Methods \\ Setting}

The University of Washington Physicians Network (UWPN) is a primary care network of 9 clinics, distributed throughout the Puget Sound region. The UWPN employs over 80 family physicians, internists, pediatricians, and mid-level providers. One unique aspect of the UWPN is the use of a electronic health record system, EPIC (Epic Systems Corporation, Madison, WI). This database captures all patient contacts including office visits, 
referrals, billing, subsequent visits, laboratory and medication orders, and visit diagnoses. With the approval and cooperation of the UWPN research committee, the UWPN information technology office provided records of all clinic encounters that took place in 1999. Telephone encounters and duplicate encounters that occurred on the same day were excluded. Encounters with providers who had fewer than 100 patient contacts were also excluded. Personal identifiers were removed from the data set. The University of Washington Institutional Review Board reviewed and approved this study.

\section{Subjects}

The unit of analysis was an individual patient encounter. Encounters, rather than individual patients, were examined to assess the independent effect of patients' comorbid conditions on the likelihood of referral.

\section{Measures}

\section{Dependent}

A referral was defined as an encounter that resulted in a consultation to another physician for specialty care.

\section{Independent}

The Andersen-Newman model of access to care, which includes predisposing, enabling, and need factors, was selected as the conceptual basis for our analyses. ${ }^{20,21}$ Predisposing factors include age, gender, race, and ethnicity. Enabling factors such as income were not available in this secondary data set. In addition, all UWPN patients have health insurance, so they are homogeneous on this enabling factor. The need factor assessed in this study is case mix.

To assess the role of burden of illness in the decision to refer we used the Johns Hopkins ACG (Adjusted Clinical Group) assignment software. $^{22,23}$ This software was developed in the 1980 s to evaluate the relationship of patient morbidity to the cost and utilization of health care services. The package assigns each patient's ICD-9-CM diagnostic codes to a unique ADG. We used the ADGs because ADGs explain more of the variation in resource use than the ACG indicators. ${ }^{4,10}$

\section{Analyses}

System level factors also influence referral patterns. For example, we observed considerable variance across the 9 study clinics in rates of referral, from 5.8 to $13.6 \%$. In addition, physicians were substantially, although not totally, nested within clinic. Accordingly, SUDAAN software (Research Triangle Institute, Chapel Hill, NC) was used to perform all analyses. Its robust variance estimator accounts implicitly for any number of stages of nesting within the primary clusters (clinics), including in this case, physicians and individual patients. Initially, bivariate tests were done to evaluate the relationship of age, gender, race, and ADG with referral. Then, individual ADGs were examined for their effect on referral likelihood after adjusting for individual patient characteristics in separate logistic regression models. We calculated "design effects" to determine how much of the variance in the estimated effects of the ADGs on referral was because of clustering: both intra-cluster correlation and cluster size. The design effect is a measure of the statistical need to account for nesting.

\section{Results}

During 1999, there were 251,240 patient encounters in the 9 clinics. Those encounters resulted in 23,720 specialty referrals, resulting in a total referral rate of 9.4 referrals per 100 encounters. Referral rates varied according to the individual patient characteristics for each encounter (Table 1). Male patient encounters had higher referral rates than female encounters (9.95 vs 9.11, $P=.02$ ). Encounters with patients $>65$ years of age and $<18$ years of age were less likely to result in a referral $(10.82$ vs 6.73 and 7.24 , respectively, $P=.001)$. Encounters with minority patients were less likely to result in a referral than encounters with white patients $(P=.002)$.

Table 1. Referral Rates by Patient Characteristics

\begin{tabular}{lrrrr}
\hline & $\mathrm{N}$ & $\begin{array}{c}\text { Percentage } \\
\text { Referred }\end{array}$ & $\chi^{2}$ & $P$ Value \\
\hline Age group & & & 33.91 & .001 \\
$\quad$ Under 18 & 62,027 & 6.73 & & \\
18 to 64 & 163,359 & 10.82 & & \\
65+ & 25,854 & 7.24 & & \\
Gender & & & 8.02 & .022 \\
$\quad$ Female & 152,269 & 9.11 & & \\
Male & 98,967 & 9.95 & & \\
Race & & & 62.81 & 0.002 \\
White & 169,020 & 9.86 & & \\
African American & 12,370 & 9.07 & & \\
Latino & 6,241 & 8.27 & & \\
Asian & 15,739 & 7.91 & & \\
$\quad$ Unknown & 17,312 & 9.24 & & \\
\hline
\end{tabular}


Adjustment for patient characteristics on comorbidity using the ACG software revealed significant predictors of referral. When analyzed independently, 21 of the 32 ADGs were significantly related with referral for any given patient encounter. When the demographic characteristics and ADGs were combined in a logistic regression model, the age and gender characteristics remained significant, and the number of significant ADGs was reduced to 19 (Table 2).

Finally, although we were not interested in the role of the clinic beyond statistically controlling for

Table 2. Adjusted Diagnostic Group (ADG) Referral Rates and Odds Ratios for Referral Controlling for Age Group, Gender, and Race

\begin{tabular}{|c|c|c|c|}
\hline $\mathrm{ADG}$ & $\begin{array}{c}\text { Referral } \\
\text { Rate }\end{array}$ & $\begin{array}{l}\text { Odds } \\
\text { Ratio }\end{array}$ & (95\% C.I.) \\
\hline \multicolumn{4}{|l|}{ Time limited } \\
\hline Minor & 9.30 & 1.01 & $(0.96,1.06)$ \\
\hline Minor-primary infections & $7.99^{* * *}$ & 0.76 & $(0.71,0.82)$ \\
\hline Major & $11.13^{*}$ & 1.19 & $(1.07,1.33)$ \\
\hline Allergies & 8.55 & 0.89 & $(0.77,1.02)$ \\
\hline Asthma & 9.30 & 0.97 & $(0.87,1.08)$ \\
\hline \multicolumn{4}{|l|}{ Likely to recur } \\
\hline Discrete & $8.21^{*}$ & 0.85 & $(0.72,1.01)$ \\
\hline Discrete infections & $11.13^{* * *}$ & 1.24 & $(1.15,1.33)$ \\
\hline Progressive & $8.19^{* *}$ & 0.86 & $(0.78,0.94)$ \\
\hline \multicolumn{4}{|l|}{ Chronic medical } \\
\hline Stable & 8.97 & 0.97 & $(0.73,1.31)$ \\
\hline Unstable & $10.30^{*}$ & 1.10 & $(1.02,1.18)$ \\
\hline \multicolumn{4}{|l|}{ Chronic specialty } \\
\hline Stable-orthopedic & 10.39 & 1.16 & $(1.00,1.34)$ \\
\hline Stable-ent & $16.84^{* *}$ & 1.83 & $(1.35,2.47)$ \\
\hline Stable-eye & $16.15^{*}$ & 2.03 & $(1.29,3.19)$ \\
\hline Unstable-ent & $13.33^{*}$ & 1.41 & $(1.11,1.78)$ \\
\hline Unstable-eye & $13.48^{*}$ & 1.60 & $(1.08,2.38)$ \\
\hline \multicolumn{4}{|l|}{ Injuries/adverse effects } \\
\hline Minor & 10.07 & 1.03 & $(0.93,1.14)$ \\
\hline Major & $11.61^{* * *}$ & 1.25 & $(1.14,1.38)$ \\
\hline \multicolumn{4}{|l|}{ Psychosocial } \\
\hline Time limited, not severe & $11.82^{* *}$ & 1.29 & $(1.13,1.48)$ \\
\hline Persistent/recurrent-stable & 10.20 & 0.99 & $(0.87,1.11)$ \\
\hline Persistent/recurrent-unstable & $10.87^{* *}$ & 1.11 & $(0.99,1.26)$ \\
\hline \multicolumn{4}{|l|}{ Signs/symptoms } \\
\hline Minor & 9.49 & 0.91 & $(0.82,1.00)$ \\
\hline Uncertain & $9.98^{*}$ & 1.12 & $(1.04,1.21)$ \\
\hline \multicolumn{4}{|l|}{ Signs/symptoms } \\
\hline Major & $11.07^{* *}$ & 1.29 & $(1.18,1.41)$ \\
\hline Discretionary & $10.22^{*}$ & 1.14 & $(1.07,1.22)$ \\
\hline See and reassure prevention & $11.99^{* * *}$ & 1.31 & $(1.22,1.40)$ \\
\hline Administrative & 10.33 & 1.10 & $(0.96,1.25)$ \\
\hline Malignancy & $8.53^{\text {*** }}$ & 0.79 & $(0.72,0.86)$ \\
\hline Pregnancy & $14.31^{* *}$ & 1.77 & $(1.43,2.19)$ \\
\hline Dental & 7.87 & 0.73 & $(0.56,0.95)$ \\
\hline
\end{tabular}

${ }^{*} P<.05$.

** $P<.01$

*** $P<.001$. its influence, its design effect was typically large on all predictors, indicating the need for the nested procedures invoked in the analysis. The average design effect for the ADGs that appear in Table 2 was 5.27. Thus, the SUDAAN procedure adjusted for a $>5$-fold increase in the variance of their estimated regression coefficients because of clustering.

\section{Discussion}

This study confirmed the variations in referral rates described by previous work. Identifying and measuring the strength of contributing factors is important in analyzing referral rates and the performance of systems of primary care. This study also demonstrates that data from electronic health records can be used to perform analyses of common primary care processes.

Comorbidity analysis remains an important tool in primary care research. This study shows the value of the ACG system in predicting the effect of patients' comorbid conditions on referral. One unique aspect of the study was the individual analysis of ADGs and their independent predictive utility. Although most researchers combine ADGs into the ACG system and sum the burden of disease scores, these results suggest that there is value to examining individual ADGs when there are sufficient data to do so.

In examining the results of this study, encounters with ADGs that signified urgent or unstable conditions were more likely to result in referral. Similarly, encounters with ADGs that reflected specialty oriented care were also more likely to be referred. These findings suggest that subsets of ADGs may be used as a means of monitoring trends in referral patterns.

This study also reinforces the value of multilevel analysis and modeling in examining complex processes in primary care. Many primary care research study designs introduce clustering or nesting into the data structure. For primary care, in which research studies are often conducted in multiple clinical sites or practice based research networks, multilevel analysis may prove to be a useful tool.

We thank the UWPN information systems staff for help in obtaining the data. Holly Andrilla contributed to preliminary analyses of the data. 


\section{References}

1. Nutting P, Franks P, Clancy C. Referral and consultation in primary care: do we understand what we're doing? J Fam Pract 1992;35:21-3.

2. Ludke RL. An examination of the factors that influence patient referral decisions. Med Care 1982;20: 782-96.

3. Henley ES. An analysis of referrals and referral rates. Hosp Health Serv Adm 1985;30:120-9.

4. Salem-Schatz S, Moore G, Rucker M, Pearson S. The case for case-mix adjustment in practice profiling. JAMA 1994;272:871-4.

5. Shea D, Stuart B, Vasey J, Nag S. Medicare physician referral patterns. Health Serv Res 1999;34:33148.

6. Lawler FH. Referral rates of senior family practice residents in an ambulatory care clinic. J Med Educ 1987;62:177-82.

7. Coulter A, Noone A, Goldacre M. General practitioners' referrals to specialist outpatient clinics. $\mathrm{Br}$ Med J 1989;299:304-8.

8. Brock C. Consultation and referral patterns of family physicians. J Fam Pract 1977;4:1129-37.

9. Bachman KH, Freeborn DK. HMO physicians' use of referrals. Soc Sci Med. 1999;48:547-57.

10. Franks $P$, Clancy C. Referrals of adult patients from primary care: demographic disparities and their relationship to HMO insurance. J Fam Pract 1997;45: $47-53$.

11. Franks P, Zwanziger J, Mooney C, Sorbero M. Variations in primary care physician referral rates. Health Serv Res 1999;34:323-9.

12. Forrest CB, Nutting PA, Starfield B, von Schrader S. Family physicians' referral decisions: results from the ASPN referral study. J Fam Pract 2002;51:215-22.

13. Calman N, Hyman R, Licht W. Variability in con- sultation rates and practitioner level of diagnostic certainty. J Fam Pract 1992;35:31-8.

14. Chan BT, Austin PC. Patient, physician, and community factors affecting referrals to specialists in Ontario, Canada: a population-based, multi-level modelling approach. Med Care 2003;41:500-11.

15. Forrest CB, Nutting P, Werner JJ, Starfield B, von Schrader S, Rohde C. Managed health plan effects on the specialty referral process: results from the Ambulatory Sentinel Practice Network referral study. Med Care 2003;41:242-53.

16. Zyzanski SJ, Flocke SA, Dickinson LM. On the nature and analysis of clustered data. Ann Fam Med 2004;2:199-200.

17. Killip S, Mahfoud Z, Pearce K. What is an intracluster correlation coefficient? Crucial concepts for primary care researchers. Ann Fam Med 2004;2:204-8.

18. Diez Roux AV. Investigating neighborhood and area effects on health. Am J Public Health. 2001;91: $1783-9$.

19. Diez-Roux AV. Bringing context back into epidemiology: variables and fallacies in multilevel analysis. Am J Public Health. 1998;88:216-22.

20. Andersen R, Newman JF. Societal and individual determinants of medical care utilization in the United States. Milbank Mem Fund Q Health Soc 1973;51:95-124.

21. Andersen RM. Revisiting the behavioral model and access to medical care: does it matter? J Health Soc Behav 1995;36:1-10.

22. Johns Hopkins University ACG Case Mix Adjustment System [computer program]. Version 6.0. Baltimore, MD: Johns Hopkins University School of Hygiene and Public Health; 2000.

23. Starfield B, Lemke KW, Herbert R, Pavlovich WD, Anderson G. Comorbidity and the use of primary care and specialist care in the elderly. Ann Fam Med 2005;3:215-22. 